\title{
Unusual sensory innervation of the dorsal hand and why we should bear this variation in mind
}

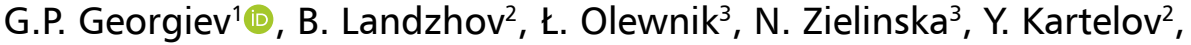 \\ I.N. Dimitrova ${ }^{4}$, R.S. Tubbs ${ }^{5-9}$ \\ 'Department of Orthopaedics and Traumatology, University Hospital Queen Giovanna - ISUL, \\ Medical University of Sofia, Bulgaria \\ ${ }^{2}$ Department of Anatomy, Histology and Embryology, Medical University of Sofia, Bulgaria \\ ${ }^{3}$ Department of Anatomical Dissection and Donation, Chair of Anatomy and Histology, \\ Medical University of Lodz, Poland \\ ${ }^{4}$ Department of Cardiology, University Hospital 'Prof. Al. Tschirkov', Medical University of Sofia, Bulgaria \\ ${ }^{5}$ Department of Anatomical Sciences, St. George's University, Grenada, West Indies \\ ${ }^{6}$ Department of Neurosurgery, Tulane University School of Medicine, New Orleans, LA, United States \\ 'Department of Neurology, Tulane University School of Medicine, New Orleans, LA, United States \\ ${ }^{8}$ Department of Structural and Cellular Biology, Tulane University School of Medicine, New Orleans, LA, United States \\ ${ }^{9}$ Department of Neurosurgery, and Ochsner Neuroscience Institute, Ochsner Health System, New Orleans, LA, United States
}

[Received: 30 September 2021; Accepted: 29 October 2021; Early publication date: 26 November 2021]

\begin{abstract}
Detailed knowledge of the anatomy and different variations of the superficial branch of the radial nerve could be of great importance not only to anatomists but also to clinicians. A predominant radial nerve supply to the dorsum of the hand is rare. Herein, we present an unusual case of unilateral sensory innervation of the dorsal hand found during routine anatomical dissection of a 72-year-old at death male Caucasian cadaver. We also present a brief discussion of the reported variation and emphasize its potential clinical implications. (Folia Morphol 2023; 82, 1: 194-197)
\end{abstract}

Key words: radial nerve, variation, dorsal hand, clinical significance

\section{INTRODUCTION}

Classically, sensory innervation of the dorsal aspect of the hand is ensured by the superficial branch of the radial nerve (SBRN), the dorsal branch of the ulnar nerve (DBUN), and the lateral antebrachial cutaneous nerve (LABCN) $[12,17]$.

The SBRN commonly curves around the wrist under the tendon of brachioradialis muscle and then divides into four or five dorsal digital branches. Usually, the first of these ensures sensory innervation of the lateral part of the thumb and the thenar eminence; it could connect with the LABCN. The second branch innervates the medial side of the thumb; the third supplies the lateral skin area of the second finger; the fourth ensures sensory innervation of the adjacent skin areas of the second and third fingers; the fifth connects to the ramus of the DBUN and supplies the neighbouring sides of the third and fourth fingers, but is often replaced by the DBUN. The dorsal digital nerves reach the root of the thumb nail, the middle phalanx of the index finger, the proximal interphalangeal joints of the third, and the lateral parts of the fourth finger. The remaining dorsal areas of the fingers are supplied by the terminal branches of the ulnar and median nerves [15].

Address for correspondence: G.P. Georgiev, MD, PhD, DSc, Department of Orthopaedics and Traumatology, University Hospital Queen Giovanna - ISUL, Medical University of Sofia, 8, Bialo More Str., BG1527 Sofia, Bulgaria, tel: +359884 493523, e-mail: georgievgp@yahoo.com

This article is available in open access under Creative Common Attribution-Non-Commercial-No Derivatives 4.0 International (CC BY-NC-ND 4.0) license, allowing to download articles and share them with others as long as they credit the authors and the publisher, but without permission to change them in any way or use them commercially. 

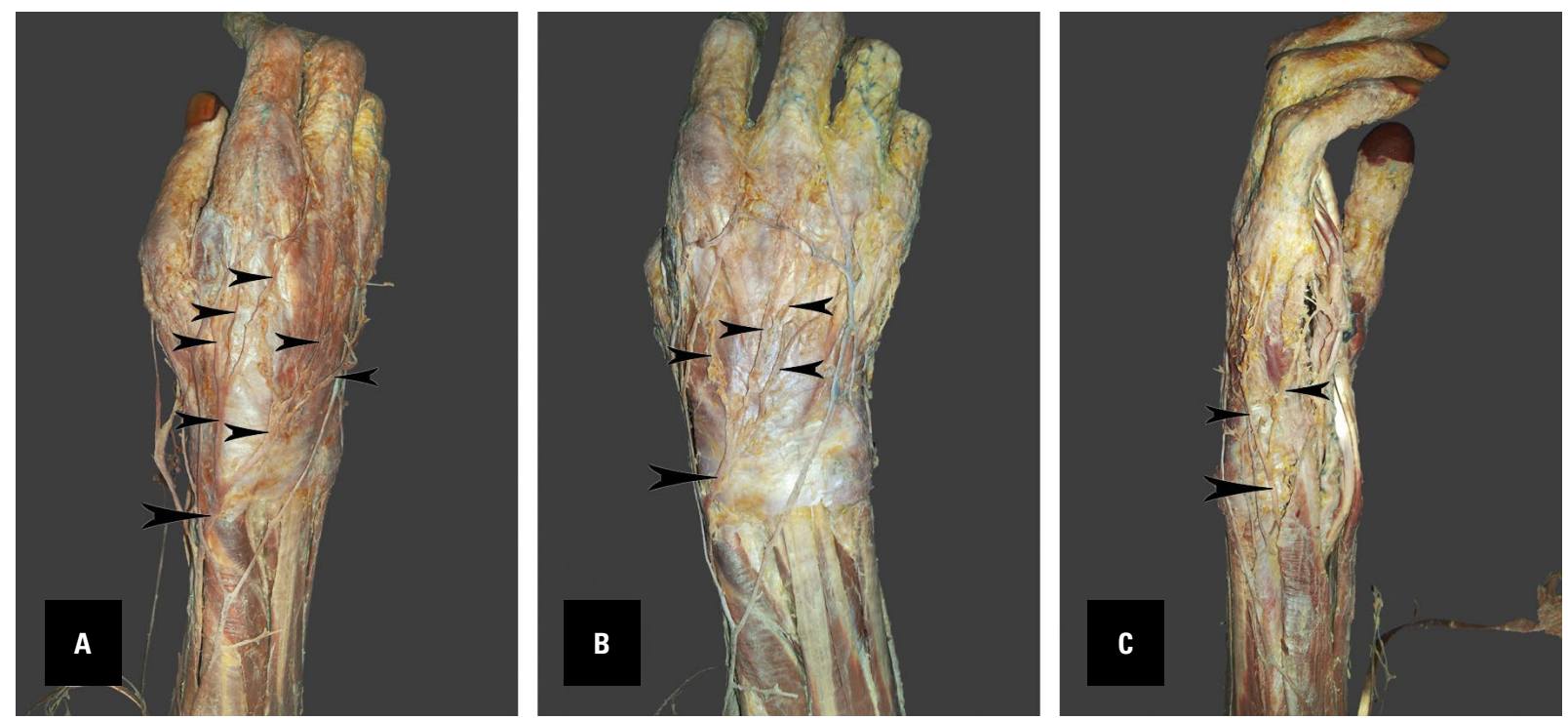

Figure 1. A-C. The lower limb of a 72-year-old at death male Caucasian cadaver showing variation of the superficial branch of the radial nerve (SBRN) and the dorsal branch of the ulnar nerve (DBUN), A, B. The large arrowhead indicates the SBRN, the small arrowhead its branches; $\mathbf{C}$. The large arrowhead indicates the DBUN, the small arrowhead its branches.

The DBUN appears near the distal part of the flexor carpi ulnaris muscle and then, after piercing the fascia, it is located on the dorsal ulnar side of the wrist and hand. Finally, it splits into two or three terminal branches; the first innervates the medial side of the fifth finger and the second the opposite sides of the fifth and fourth fingers; when there is a third branch, it ensures sensory innervation of the opposite sides of the third and fourth fingers [15].

The LABCN is a cutaneous branch of the musculocutaneous nerve. Distally, it reaches the base of the thenar area and some of its terminal branches can connect with the terminal branch of the radial nerve and the palmar cutaneous branch of the median nerve [15].

Precise knowledge of the dorsal sensory innervation of the hand is not only interesting to anatomists but also has clinical implications for hand surgeons during dorsal flaps and approaches, for regional anaesthetic blocks, and for neurological practice to avoid misinterpretation of nerve pathology [5, 17].

Herein, we present an unusual case of unilateral variation of the SBRN in the right hand.

\section{CASE REPORT}

During a routine anatomical dissection of the right upper limb of a 72-year-old male Caucasian cadaver a rare SBRN variation was observed.

After curving around the wrist under the tendon of the brachioradialis muscle, $8.7 \mathrm{~cm}$ from the radial styloid process (RS), and passing over the tendons of the abductor pollicis longus and extensor pollicis brevis muscles, the SBRN divides into two branches. The smaller branch supplies the skin of the medial and lateral sides of the thumb and reaches the root of the nail. The larger branch divides into three smaller branches: the first supplies the skin of the dorsal hand and adjoining sides of the index finger (the medial branch of the nerve to the finger was damaged during the dissection course); the second divides into a further two branches, and the medial one also divides into two, so the major branch ensures innervation of the middle and ring fingers to the proximal interphalangeal joints; and the third supplies the lateral surface of the little finger (Fig. 1A, B; 2).

The DBUN appears near the flexor carpi ulnaris muscle in the distal direction, and after piercing the fascia passes to the ulnar side on the dorsal part of the wrist and hand and finally divides into two branches: the first innervates the medial side of the little finger and the second communicates with a ramus of the superficial branch of the ulnar nerve and thus supplies the skin of the medial palmar side of the fifth finger (Fig. 1C; 2 ).

\section{DISCUSSION}

Several previous articles have presented different innervation variants of the dorsal part of the hand [12].

Mok et al. [12] observed that the common innervation distribution pattern in more than half of all 


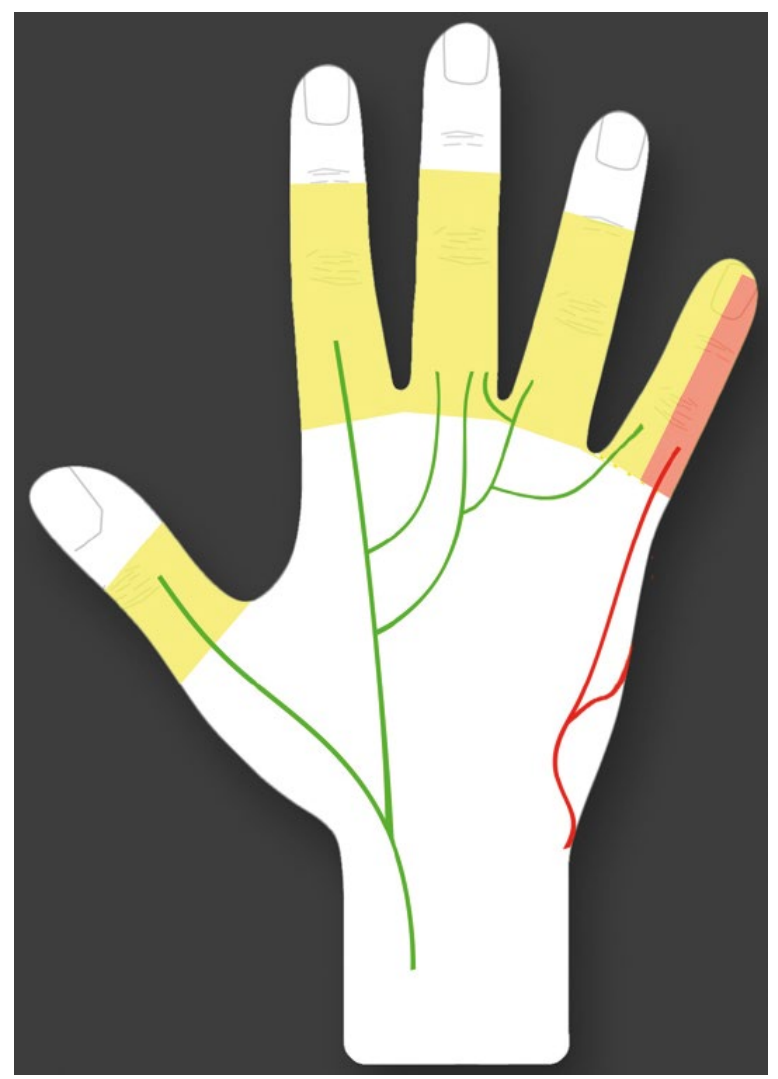

Figure 2. Scheme showing variation of the superficial branch of the radial nerve (green line) and the dorsal branch of the ulnar nerve (red line).

cases was distributed equally between the SBRN and the DBUN. Vergara-Amador and Nieto (2010) [19] described similar results in $56 \%$ of cases. Linell [9] reported that in $68.8 \%$, the DBUN supplies the dorsal parts of the medial two and a half digits. According to Mok et al. [12] the nerve territories overlapped significantly among different specimens. This agrees with the findings of other authors $[2,6,11,13]$.

Sulaiman et al. [17] established that the SBRN innervated the thumb and index finger in $63.6 \%$ of cases. The DBUN was described as extending the innervation zone more laterally, ensuring the supply to the skin of the ulnar part of the second finger in 3 cases. The usual innervation pattern of the dorsal hand was found in only $12.86 \%$ of the hands examined. Sulaiman et al. [17] established that the radial nerve is the sole supplier to the dorsum of the hand in $6.62 \%$ of cases when the DBUN is absent. Similar results were reported by Botte et al. [3] in 4.2\%, Mok et al. [12] in 3.3\%, Robson et al. [14] in $8 \%$ and Tiznado et al. [18] in 5.6\%. Kuruvilla et al. [7] noted an autosomal dominant inheritance of a variant SBRN that predominantly innervated the dorsal aspect of the hand. They reported a patient with such a bilateral variation and a similar one in one of the two children.

Sulaiman et al. [17] also reported that the ulnar part of the fifth finger was supplied by the DBUN in all cases when the nerve was established. The DBUN supplied the lateral side of the little finger in $97.9 \%$ and the medial side of the ring finger in $96.4 \%$. It provided the sole innervation of the lateral side of the ring finger in $63.6 \%$. The reported variation, if it compressed or injured the ulnar nerve, could present as loss of sensation on the dorsal surface of the medial part of the fifth finger.

Mok et al. [12] proposed a classification of the nerve distribution of the dorsal hand, termed the radial/ulnar/lateral antebrachial cutaneous nerve (RUL) classification system. They accept the normal anatomical pattern as classically described: the SBRN innervates the radial two and a half digits, the DBUN, the ulnar two and a half, and the LABCN innervates no digits and is used for the term Rn/Un/Ln (" $n$ " for normal). When presented in different variants, each nerve is described separately. The described nerve is assigned +1 or -1 for each half of the finger that has additional or less innervation [12].

The sensory innervation differs between the two hands. The symmetry in innervation of the dorsal hand ranges between $29 \%$ [16] and $43.2 \%$ [17]. Therefore, Sulaiman et al. [17] considered that clinicians should be very careful when they compare healthy with diseased hands in nerve conduction studies.

Clinically, variations of the SBRN involving all or most of the supply to the dorsal part of the hand could impede hand and reconstructive surgeons, and cause difficulty for neurologists interpreting electromyograms or evaluating abnormal symptoms. The presented variation could cause problems for hand and wrist surgery through the dorsal approach, and no safe zone could be defined. In addition, in the event of local block of the SBRN and anaesthesia of the fifth finger, a surgeon should be suspicious for possible SBRN variations, and precise operative technique is mandatory $[1,7,12,17,18]$. Moreover, in the event of traumatic injury to the ulnar nerve, such a SBRN variation could lead to misdiagnosis [8].

Auerbach et al. [2] established that the SBRN appeared $8.6 \mathrm{~cm}$ proximal to the RS between the tendons of the branchioradialis and extensor carpi radialis longus. This nerve then appeared $6.0 \mathrm{~cm}$ 
above the fascia from the RS. In the series of Vergara-Amador and Nieto [19] it appeared subcutaneously $8.45 \mathrm{~cm}$ from the RS. Mok et al. [12] established that the SBRN pierced the fascia between the tendons of the aforementioned muscles $8.7 \mathrm{~cm}$ from the RS. These authors recommend that block of the SBRN should be $7 \mathrm{~cm}$ proximal to the radial styloid, where all its branches perforate the fascia and the nerve is widest. However, Mackinnon and Dellon [10] point out that the supply from the $L A B C N$ also needs to be borne in mind because it could overlap with these nerves.

\section{CONCLUSIONS}

The possibility of a variant total or predominant supply of the dorsal skin by the radial nerve, as reported, could explain atypical symptoms in entrapment neuropathies or acute nerve injury, and could impede electrophysiological tests and dorsal approaches to the hand. As stated by Żytkowski et al. [20] the analysis of such a variation contributes for obtaining an actual, not idealized image of the inside of the human body, which is of crucial importance in everyday clinical practice.

\section{Acknowledgements}

The authors wish to express their gratitude to all those who donated their bodies to medical science [4].

\section{Conflict of interest: None declared}

\section{REFERENCES}

1. Abrams RA, Brown RA, Botte MJ. The superficial branch of the radial nerve: an anatomic study with surgical implications. J Hand Surg Am. 1992; 17(6): 1037-1041, doi: 10.1016/ s0363-5023(09)91056-5, indexed in Pubmed: 1430933.

2. Auerbach DM, Collins ED, Kunkle KL, et al. The radial sensory nerve. An anatomic study. Clin Orthop Relat Res. 1994(308): 241-249, indexed in Pubmed: 7955689.

3. Botte MJ, Cohen MS, Lavernia CJ, et al. The dorsal branch of the ulnar nerve: an anatomic study. J Hand Surg Am. 1990; 15(4): 603-607, doi: 10.1016/s03635023(09)90022-3, indexed in Pubmed: 2380524.

4. Iwanaga J, Singh V, Ohtsuka A, et al. Acknowledging the use of human cadaveric tissues in research papers: Recommendations from anatomical journal editors. Clin Anat. 2021; 34(1): 2-4, doi: 10.1002/ca.23671, indexed in Pubmed: 32808702.

5. Keplinger M, Marhofer P, Moriggl B, et al. Cutaneous innervation of the hand: clinical testing in volunteers shows high intra- and inter-individual variability. $\mathrm{Br} \mathrm{J}$ Anaesth. 2018; 120(4): 836-845, doi: 10.1016/j.bja.2017.09.008, indexed in Pubmed: 29576124.
6. Kosinski C. L'innervation cutanée de la face dorsale de la main, basée sur l'examen de 300 pièces anatomiques, avec quelques notions d'anatomic comparée. Assoc Anatom Compt Rend. 1927; 22: 121-133.

7. Kuruvilla A, Laaksonen S, Falck B. Anomalous superficial radial nerve: a patient with probable autosomal dominant inheritance of the anomaly. Muscle Nerve. 2002; 26(5): 716-719, doi: 10.1002/mus.10239, indexed in Pubmed: 12402296

8. Leis AA, Wells KJ. Radial nerve cutaneous innervation to the ulnar dorsum of the hand. Clin Neurophysiol. 2008; 119(3): 662-666, doi: 10.1016/j.clinph.2007.11.045, indexed in Pubmed: 18164664.

9. Linell EA. The distribution of nerves in the upper limb, with reference to variabilities and their clinical significance. J Anat. 1921; 55(Pt 2-3): 79-112, indexed in Pubmed: 17103922.

10. Mackinnon SE, Dellon AL. The overlap pattern of the lateral antebrachial cutaneous nerve and the superficial branch of the radial nerve. J Hand Surg Am. 1985; 10(4): 522-526, doi: 10.1016/50363-5023(85)80076-9, indexed in Pubmed: 4020063.

11. Mogi E. Untersuchung über die sensible innervation der handrücken bei den japanischen feten. Redaktion Der Okijimas Folia Anat Jap. 1937; 15: 675.

12. Mok D, Nikolis A, Harris PG. The cutaneous innervation of the dorsal hand: detailed anatomy with clinical implications. J Hand Surg Am. 2006; 31(4): 565-574, doi: 10.1016/j.jhsa.2005.12.021, indexed in Pubmed: 16632049 .

13. P'an MT. The cutaneous nerves of the Chinese hand. Am J Phys Anthropol. 1939; 25(3): 301-309, doi: 10.1002/ ajpa.1330250326.

14. Robson AJ, See MS, Ellis H. Applied anatomy of the superficial branch of the radial nerve. Clin Anat. 2008; 21(1): 38-45, doi: 10.1002/ca.20576, indexed in Pubmed: 18092362 .

15. Standring S. Gray's anatomy: the anatomical basis of clinical practice. Churchill Livingstone/Elsevier, Edinburgh 2008.

16. Stappaerts KH, Van Hees J, Van den Broeck EA. Peripheral cutaneous nerve distribution to the fingers. Physiother Res Int. 1996; 1(1): 41-49, doi: 10.1002/pri.46, indexed in Pubmed: 9238722.

17. Sulaiman $S$, Soames $R$, Lamb C. The sensory distribution in the dorsum of the hand: anatomical study with clinical implications. Surg Radiol Anat. 2015; 37(7): 779-785, doi: 10.1007/s00276-014-1416-1, indexed in Pubmed: 25572036 .

18. Tiznado G, Sousa-Rodrigues C, Olave E. Superficial branch of the radial nerve: large distribution in the dorsum of hand. Int J Morphol. 2012; 30(2): 374-378.

19. Vergara-Amador E, Nieto JL. Estudio anatómico de la rama superficial del nervio radial, implicaciones quirúrgicas. Rev Fac Med. 2010; 58(3): 214-220.

20. Żytkowski A, Tubbs R, Iwanaga J, et al. Anatomical normality and variability: Historical perspective and methodological considerations. Transl Res Anat. 2021; 23: 100105, doi: 10.1016/j.tria.2020.100105. 